\title{
Políticas que favorecen la inclusión
}

\section{Ángel Elías Ortega}

Facultad de Relaciones Laborales y Trabajo Social, Universidad del País Vasco (UPV/EHU)

<angel.elias@ehu.eus>

\section{Leire Rincón García}

Universidad de Barcelona

<lrincon@ibei.org>

Edozein gizarteratze-politikarako ezinbestekoa gertatzen da gizartearen ahalegin eta ondareak birbanatzea. Gaur eguneko munduan aurrerapen teknologikoei esker aberastasuna ugaltzen den bitartean, enpleguak gutxitu egiten dira, eta premiazkoa da gizartean bizi den ororen artean modu justu batean banatzea bai aberastasuna bai enpleguari eskainitako orduen kopurua. Horretarako, behar-beharrezko gertatzen dira oinarrizko errenta, enplegu-lanaldiaren murrizketa eta hezkuntza inklusiboa.

\section{GAKO-HITZAK:}

Aberastasunaren banaketa, enplegua, lanaldiaren murrizketa, oinarrizko errenta, hezkuntza inklusiboa.
Cualquier política de inclusión exige una sociedad que comparta los esfuerzos y las ganancias. En el actual mundo de grandes avances tecnológicos, que derivan en la supresión de empleos y el incremento exponencial de bienes, es necesario que las riquezas y el tiempo del empleo se repartan de forma justa y equitativa entre todas las personas de la sociedad. A ello deben contribuir la renta básica y la reducción de la jornada laboral, así como una educación inclusiva.

\section{Palabras Clave:}

Reparto de la riqueza, empleo, reducción de la jornada laboral, renta básica, educación inclusiva. 


\section{Introducción}

Para reflexionar sobre las necesidades, las políticas y las intervenciones en el ámbito de la inclusión social e intentar que sea de utilidad en la elaboración del IV Plan Vasco de Inclusión Social Activa, queremos empezar nuestro artículo con una mención al anterior Plan. El III Plan Vasco de Inclusión Social Activa se encuentra estructurado en nueve objetivos estratégicos, pero ninguno aboga por un cambio de estructuras que corrija las grandes desigualdades en el reparto de la riqueza. Más bien pone el centro de mira en las personas vulnerables y confía en el empleo como el instrumento válido para la inclusión social. Así, el primero de sus objetivos habla de “impulsar la inclusión social eficaz mediante la activación desde el empleo a las personas más vulnerables para reducir la pobreza y la exclusión social, asegurando un nivel de renta digno y el acceso de las personas empleables a un empleo decente".

Creemos que cualquier Plan Vasco de Inclusión debe exigir, en primer término, hacer frente a las estructuras y factores que acentúan la vulnerabilidad de las personas de la comunidad autónoma. Desde hace mucho tiempo, sabemos que el crecimiento económico no es garantía de un adecuado reparto y bienestar social, y en el caso de la Comunidad Autónoma Vasca (CAV), Confebask prevé para 2017 un crecimiento de la economía del 2,5\%, cinco décimas menos que en 2016 ${ }^{1}$. Sin embargo, como veremos a continuación, la riqueza no está bien repartida y el empleo decente no llega a todas las personas, pues en bastantes casos las condiciones laborales son precarias. Por ello, de forma sucesiva, abogaremos por una estrategia que tenga como objetivos principales conseguir una reducción de la jornada laboral que permita un empleo para toda la población y en un reparto justo de la riqueza que consagre el derecho a una renta básica universal con un importe equivalente al umbral de la pobreza. Pero, también, en una educación inclusiva que se base en las capacidades de cada individuo para motivarle en su permanente desarrollo como persona.

\section{Algunos pocos datos sobre el reparto de la riqueza}

Somos una sociedad global en la que tan sólo 8 personas tienen tanto como la mitad del mundo, 3.600 millones. Una economía para el $99 \%$, el nuevo informe de Oxfam-Intermón (Macías y Ruiz Rodríguez, 2017), refleja cómo la súper concentración de riqueza en el mundo se ha agudizado en este último año, amenazando la estabilidad y el crecimiento mundial. En el último año, ha habido 7.000 nuevos millonarios en España, y la fortuna de tan sólo 3 personas

${ }^{1}$ En su Informe de Coyuntura Económica, de diciembre de 2016, Confebask analiza las perspectivas para 2017, planteando retos y recomendaciones. Sin embargo, en lugar alguno hace referencia a la necesidad de corregir las elevadas desigualdades económicas. equivale ya a la riqueza del $30 \%$ más pobre del país, es decir, de 14,2 millones de personas. Mientras que en 2015 este $30 \%$ más pobre vio reducida su riqueza en más de una tercera parte (-33,4\%), la fortuna de las tres personas más ricas del país aumentó un $3 \%^{2}$. Tal como señala el citado informe, las explicaciones a esta gravísima desigualdad hay que buscarlas en un modelo económico donde los beneficios empresariales son en gran medida a costa de peores salarios y de un sistema tributario que no grava suficientemente a quienes más tienen, siendo muy tolerante con el fraude y los paraísos fiscales 3 . Éste es un fenómeno que va en aumento, ya que en la última década la recaudación de impuestos a sociedades ha disminuido casi un $50 \%{ }^{4}$. Además, el peso fiscal ha recaído de manera creciente sobre el consumo y el trabajo. Por ello, es importante poner el foco no tanto en las personas más necesitadas, sino en las instituciones -entendidas no sólo como organizaciones, sino también como normasque permiten que se perpetúen y aumenten las dinámicas de desigualdad y explotación. Así, se hace indispensable para la inclusión social incluir a aquellos sectores y personas que, por sus creencias o actividades, perpetúan las dinámicas de exclusión.

Sin duda, esta desigualdad aumenta el riesgo de pobreza y de exclusión, ya que las oportunidades no son iguales para todas las personas. Así, la Encuesta de Pobreza y Desigualdades Sociales de la CAV de 2014 señalaba que 56.307 personas $(27,1 \%$ del total en riesgo de pobreza) no fueron atendidas por el sistema de rentas mínimas ${ }^{5}$ y que un $9 \%$ de las niñas y niños en el País Vasco viven en situación de pobreza extrema (Alonso y Marcos, 2017: 16).

Como viene señalando el ecologismo, la economía no puede seguir obviando por más tiempo los límites físicos del planeta, lo que exige un nuevo modelo productivo con pautas de crecimiento de la ocupación laboral que se dirijan hacia sectores que minimicen

2 Oxfam-Intermón publica anualmente un informe sobre la distribución de la riqueza, tanto a escala mundial como para España, aunque todavía no existe un adecuado desglose por comunidades autónomas. El nํㅜ 42, publicado en enero de 2017 , es el último hasta la fecha.

${ }^{3}$ La filtración de los 'papeles de Panamá' volvió a poner en la palestra el asunto de los paraísos fiscales. Según la Red para la Justicia Global (Tax Justice Network, 2016), en 2015 había unos 26 billones de euros (32 trillones de dólares) ocultos y libres de impuestos en los diversos paraísos fiscales, lo que supone un tercio del PIB mundial, que asciende a 75 billones. A su vez, el Informe sobre economía sumergida y fraude fiscal en Euskadi, elaborado por los catedráticos del Instituto de Economía Pública de la UPV/EHU Ignacio Zubiri y Javier Fernández-Macho, y por la catedrática Mari Carmen Gallastegui, apunta a que en el periodo 1990-2014 la economía sumergida de la Comunidad Autónoma Vasca fue de entre el $17 \%$ y el $17,4 \%$ del PIB, siempre por debajo de la estimada en la mayoría de estudios para España. El diferencial se sitúa en torno a los 5,5 puntos porcentuales durante el primer periodo de la crisis económica (2007-2010) y entre el $4,3 \%$ y el $5 \%$ en el cuatrienio más reciente (Departamento de Hacienda y Economía, 2016).

${ }_{4}$ Así aparece en el informe Beneficios para quién, de Oxfam-Intermón (2016), que señala los paraísos fiscales como principal amenaza para una fiscalidad justa.

5 Nos referimos a tres clases de ayudas no contributivas que rigen en la Comunidad Autónoma del País Vasco: la renta de garantía de ingresos (RGI), la prestación complementaria de vivienda (PCV) y las ayudas de emergencia social (AES). 
los efectos irreversibles al medio ambiente. En buena medida, estos sectores se corresponden con necesidades sociales y ambientales insatisfechas.

Precisamente en la línea del Pacto Mundial de Naciones Unidas y de las recomendaciones de la Comisión Europea, el Centro Internacional de Investigación e Información sobre la Economía Pública, Social y Cooperativa de España (CiriecEspaña) acaba de crear una web ${ }^{6}$ que desea contribuir al profundo debate global sobre la necesidad de un cambio de paradigma económico hacia fórmulas que, en su desempeño, tengan en cuenta parámetros mucho más allá de la rentabilidad económica, como son la dignidad de las personas, el respeto de las comunidades, y la protección del medio ambiente y lucha contra el cambio climático. También en la Comunidad Autónoma Vasca existe el Instituto de Derecho Cooperativo y Economía Social/ Gizarte-Ekonomia eta Zuzenbide Kooperatiboaren Institutua (Gezki)7, que, entre otros logros, ha conseguido la puesta en marcha del Observatorio Vasco de Economía Social ${ }^{8}$, el cual permite la identificación y cuantificación de la actividad de la economía social de la Comunidad Autónoma del País Vasco, entendiendo ésta como el sector que aglutina a aquellas entidades de naturaleza privada cuya característica común es que se constituyen para satisfacer necesidades sociales y no para retribuir a inversores capitalistas.

Por ello, proponemos la adopción de una estrategia que persiga la creación de una economía solidaria, que esté al servicio de la comunidad y cumpla los seis principios de la Carta de la Economía Solidaria: equidad, trabajo, sostenibilidad ambiental, cooperación, sin fines lucrativos y compromiso con el entorno?. $\operatorname{dos} />$.

${ }^{6}$ Disponible en $<$ http://www.economiasostenible.org/bienveni7 .

7 El 8 de mayo de 1987 fue aprobada su creación en el seno de la UPV/EHU. Su página web es 〈http://www.gezki.eus〉.

${ }^{8}$ El Observatorio Vasco de Economía Social nace al amparo del convenio de colaboración de 30 de diciembre de 2008 firmado entre el Gobierno Vasco, representado por el consejero de Justicia, Empleo y Seguridad Social, y la UPV/EHU, representada por el Instituto de Derecho Cooperativo y Economía Social-Gezki. Está disponible en 〈http://www.oves-geeb.com/es/observatorio〉.

9 Las organizaciones que participan en el movimiento de la economía solidaria, en general, y en REAS-Red de Redes, en particular, comparten, para el desarrollo de su misión, la Carta de Principios de la Economía Solidaria (2011), que tiene los siguientes ejes transversales:

- La autonomía como principio de libertad y ejercicio de la corresponsabilidad.

- La autogestión como metodología que respeta, implica, educa, iguala las oportunidades y posibilita el empoderamiento.

- La cultura liberadora como base de pensamientos creativos, científicos y alternativos que nos ayuden a buscar, investigar y encontrar nuevas formas de convivir, producir, disfrutar, consumir y organizar la política y la economía al servicio de todas las personas.

- El desarrollo de las personas en todas sus dimensiones y capacidades: físicas, psíquicas, espirituales, estéticas, artísticas, sensibles, relacionales... en armonía con la naturaleza, por encima de cualquier crecimiento desequilibrado económico, financiero, bélico, consumista, transgénico y anómalo como el que se está propugnando en nombre de un desarrollo 'ficticio'.

- La compenetración con la Naturaleza.

\section{Situación del empleo}

Hay que empezar por recordar que empleo y trabajo no son lo mismo. El trabajo se define ${ }^{10}$ como el "conjunto de actividades, remuneradas o no, cuyos resultados proporcionan bienes y servicios a los miembros de nuestra especie y sirven para su reproducción", mientras que el empleo se reduce a las actividades que reciben contraprestación económica. Por tanto, la diferencia fundamental entre trabajo y empleo es la gran separación entre la esfera pública o formal, donde se sitúa el trabajo productivo, y la esfera privada o informal, donde se sitúa el trabajo reproductivo ${ }^{11}$.

Si vemos los últimos datos del mercado laboral en el País Vasco, comprobamos que:

- Euskadi tiene, tras el segundo trimestre de 2017, 114.300 personas desempleadas (un $52 \%$ de ellas, mujeres), lo que significa una tasa de paro del 11,23\%. Sin embargo, el empleo no ha crecido, ya que las tasas de paro ${ }^{12}$ caen, en parte debido a la pérdida de población activa ${ }^{13}$, así:

- Respecto al final del primer semestre de 2016, el empleo estimado en la CAV ha aumentado en 10.800 personas, mientras que la afiliación ha disminuido en 25.039 .

- La suma de personas ocupadas y paradas da como resultado que la población activa en Euskadi era, en el primer trimestre de 2017, la menor de los últimos nueve años, al disminuir en 12.500 personas durante el último año y perder casi 90.000 personas desde 2011.

- Ocurre lo mismo entre la población juvenil, que, comparando con el máximo alcanzado en 2009, ha descendido en 42.300 personas.

\footnotetext{
- La solidaridad humana y económica como principio de nuestras relaciones locales, nacionales e internacionales.

${ }^{10}$ Entre otras muchas voces, así lo definen Raventós y Wark
} (2016).

${ }^{11}$ Se ha escrito mucho sobre el trabajo reproductivo, pero por su claridad, precisión y concisión, recomiendo el artículo de Carrasquer et al. (1998). La incorporación de la mujer al mundo laboral supuso toda una revolución social en el proceso de emancipación femenina. Sin embargo, además de que esa emancipación está todavía lejos de ser completa, se ha caído en una nueva trampa: 'la doble presencia' o la doble jornada de trabajo que realizan las mujeres trabajadoras en la empresa y en el hogar, sobre la que realizó su tesis doctoral la ya citada socióloga Carrasquer (2009).

${ }^{12}$ Como sostiene Felgueroso (2014), la medición estándar y oficial de paro - establecida bajo los criterios de la Organización Internacional del Trabajo- es manifiestamente insuficiente: por un lado, no considera a las personas desanimadas como paradas; tampoco incluye a las que no buscan empleo, pero desean trabajar; y, por último, no toma en cuenta el paro a tiempo parcial o subempleo. Estas críticas sí son consideradas en la estadística oficial de los Estados Unidos, donde el Bureau of Labor Statistics mide seis tipos distintos de paro, siendo para nuestros intereses los cuatro últimos los que reflejan esta crítica.

${ }^{13}$ Según el Informe Laboral Euskadi correspondiente al segundo trimestre de 2017 (De la Rica, Demel y Miller, 2017: 2), se ha producido "un aumento total en la población de 5.600 adultos en los últimos doce meses. Este cambio neto se descompone desde el punto de vista laboral en un incremento de 2.800 ocupados, un aumento de 18.100 inactivos y un descenso de 15.300 personas desempleadas". 
- El $63 \%$ de las personas desempleadas llevan más de un año buscando empleo y la tasa de temporalidad de las personas ocupadas es del $24 \%$, que es el tercer peor dato de los 28 socios de la Unión Europea, sólo por detrás de Polonia, que tiene el $26,7 \%$, y del conjunto del Estado español, que llega al $26,5 \%$.

- Los puestos de trabajo que se han generado son cada vez más precarios, debido al aumento de la temporalidad, los contratos a tiempo parcial y la disminución de su duración. En concreto, durante el primer trimestre de 2017 (Servicio Público de Empleo Estatal, cit. en Instituto Manu RoblesArangiz, 2017: 5):

- De los 100.000 contratos que al mes se firman en Euskadi, el $36,4 \%$ son a jornada parcial, y el $91,3 \%$, temporales.

- Casi la mitad de los contratos, el 45,7\%, duran menos de un mes, y el $11,8 \%$ son de menos de una semana.

\section{Reducción de la jornada laboral}

Pero si ésa es la foto actual, el Foro Económico Mundial ${ }^{14}$ pone cifras a las consecuencias que la robótica y las nuevas tecnologías van a tener en el empleo, dado que a escala mundial se destruirán 5,1 millones de puestos de trabajo netos entre 2015 y 2020. A su vez, el dictamen del Comité Económico y Social Europeo (2016) concluye que, si bien la digitalización producirá ganancias de productividad, no se conoce con precisión su efecto sobre los niveles de empleo, aunque está claro que la tendencia futura será negativa para el mercado laboral y la organización del trabajo. En el contexto español, la consultora Adecco, a través de una encuesta a personas expertas en recursos humanos (Opinno, 2016), afirma que en el futuro las consecuencias más importantes sobre el empleo en España derivarán de los avances tecnológicos, con 4,8 puntos en una escala de 5 , y que entre esos avances tecnológicos, la robotización del trabajo tendrá un efecto sustantivo (3,85 puntos en una escala de 5).

La lucha por una jornada laboral que permita trabajar para vivir y no vivir para trabajar es indisociable del movimiento obrero, como lo acredita la celebración anual del 1을 de mayo como el Día Internacional de las y los Trabajadores a causa de la Matanza de Chicago de $1886^{15}$. En el siglo XIX, este objetivo determinó

${ }^{14}$ Según el Foro Económico Mundial en su informe The Future of Jobs (2016), "La cuarta revolución industrial ya está aquí y, como no espabilemos, su impacto social va a ser mayor que el de todas las anteriores". La trascendencia del asunto ha hecho que el borrador elaborado por la Unión Europea con las convocatorias para el Programa del Reto Social 6 de Horizon 2020 (periodo 2018-2020), 'Europa en un mundo cambiante: sociedades inclusivas, innovadoras y reflexivas', incluya, dentro de la convocatoria 'Transformaciones socioeconómicas de la cuarta revolución industrial', un área de estudio referida a 'La investigación para el crecimiento inclusivo: los efectos socioeconómicos de las transformaciones tecnológicas'.

${ }^{15}$ Lugar y fecha en la que obreros anarquistas reivindicaron la jornada laboral de ocho horas: ocho horas para el trabajo, ocho horas gran parte de las reivindicaciones obreras ${ }^{16} \mathrm{y}$, en un primer momento, las medidas de reducción de la jornada se inspiraron en la necesidad de mejorar la seguridad y salud de los trabajadores. El Tratado de Versalles, que puso fin a la Primera Guerra Mundial, recomendaba la jornada de 8 horas diarias, las 48 semanales y que los domingos fuesen festivos, propuestas que fueron recogidas por la Organización Internacional del Trabajo (OIT) en 1919, con el añadido de que se librase la tarde de los sábados. Por su parte, en España no fue hasta el Decreto de 3 de abril de 1919 que se aprobó la jornada de 8 horas diarias y 48 horas a la semana ${ }^{17}$. Desde 1960 , la jornada laboral está fijada en 8 horas diarias y 40 horas a la semana. Esta ligera reducción de horas semanales contrasta gravemente con el dato de que, en este mismo periodo, se haya producido un enorme incremento de la producción y de la productividad, pues para producir lo que 100 personas en la España de 1960, se necesitaban en 1995 únicamente 27 personas y 24,4 personas en $2012^{18}$.

La limitación de las horas de trabajo se ha expresado de forma mayoritaria mediante la fijación de límites a la jornada semanal, como acredita el gran número de países que han reducido la jornada semanal de 48 a 40 horas en las últimas décadas ${ }^{19}$. Así, en

para el reposo y ocho horas para la educación, y fueron asesinados por la Policía al manifestarse.

${ }^{16} \mathrm{El}$ primero en reivindicar la jornada laboral de ocho horas fue el socialista utópico Robert Owen en 1817, pero fue en el Manifiesto Inaugural de la I Internacional (1864) redactado por Karl Marx, donde aparece reflejada su importancia: "[En la] lucha por la limitación legal de la jornada de trabajo [...] de lo que se trataba era de decidir la gran disputa entre la dominación ciega ejercida por las leyes de la oferta y la demanda, contenido de la Economía política burguesa, y la producción social controlada por la previsión social, contenido de la Economía política de la clase obrera. Por eso, la ley de la jornada de diez horas no fue tan sólo un gran triunfo práctico, fue también el triunfo de un principio; por primera, vez la Economía política de la burguesía había sido derrotada en pleno día por la Economía política de la clase obrera". Dos años después, en 1866, el propio Marx convirtió la limitación de la jornada laboral en una condición necesaria para la emancipación de la clase obrera. Visto cronológicamente, en 1802 se consiguió la limitación de 12 horas diarias para el trabajo infantil; en 1830 , una huelga de hasta 100.000 obreros reclamó la reducción de jornada de 16 horas a 10 horas diarias. En 1857, se consiguió limitar la jornada a las 10 horas diarias, pero sólo para varones menores de edad y mujeres. Y en 1886, en Chicago, tuvo lugar la mencionada reclamación de la jornada de ocho horas.

${ }_{17} \mathrm{El}$ desenlace final de esta conquista se produjo con la huelga de La Canadiense, iniciada con el despido de 8 obreros sindicalizados de una compañía eléctrica de Barcelona y secundado posteriormente por el sindicato CNT, que terminó convirtiéndose en una gran huelga general que paralizó durante 44 días el $70 \%$ de la industria catalana. Hasta esa fecha, la jornada de 8 horas diarias y 48 horas a la semana sólo era realizada por el $17,8 \%$ de los obreros, y su progresivo cumplimiento requirió de un elevado número de actas de infracciones a las empresas realizadas por los inspectores de trabajo, tal como señala Soto (1989: 612-615).

${ }^{18}$ Cálculos realizados en el marco de la investigación sobre la necesidad y viabilidad de las políticas de reducción de tiempos de trabajo por Castrillón et al. (2015), siguiendo la metodología previamente utilizada por Montes (1996: 82).

${ }^{19}$ La $19^{\text {a }}$ Conferencia Internacional de Trabajo, reunida en Ginebra el 4 de junio de 1935 , ya constataba que "el desempleo se ha extendido tanto y se ha hecho tan persistente que en la actualidad millones de trabajadores, sin ser responsables de su situación, están en la miseria y sufren privaciones de las que legítimamente tienen derecho a ser aliviados"; y consideraba que, "para dar efecto a las resoluciones adoptadas por las reuniones decimoctava y decimonovena de la Conferencia Internacional del Trabajo, es indispensable desplegar un esfuerzo continuo a fin de reducir lo más posible las 
2009 el $41 \%$ de los Estados tienen establecida la jornada de 40 horas semanales, entre ellos, el $67 \%$ de los países desarrollados y de la Unión Europea. Del $44 \%$ cuya jornada regular supera las 40 horas, más de la mitad ha fijado un límite de 48 horas $^{20}$. Sin duda, de especial interés es el caso de Francia con las Leyes Aubry (1998-2000) y, especialmente, la obligación de reducción de jornada de trabajo a 35 horas semanales en todas las empresas del sector mercantil de más de 20 asalariados a partir del 1 de enero de 2000 , cuyos resultados corroboró un informe de la comisión de investigación de la Asamblea Nacional Francesa de 2014: entre 1999 y 2000 , periodo en que se aplicó la Ley a grandes empresas y al sector público, se crearon 350.000 empleos directos gracias a esta medida ${ }^{21}$.
En la actualidad, se está poniendo en práctica en el sur de Europa una reducción del tiempo de trabajo mediante el trabajo a tiempo parcial. La contratación a tiempo parcial tiene unas características muy negativas y, junto con los contratos temporales, se considera que constituye un supuesto de baja calidad del empleo. Este aspecto se acentúa por la creciente involuntariedad del contrato a tiempo parcial, ya que en una elevada proporción se acepta forzosamente en defecto de uno a jornada completa $^{22}$. A pesar del incremento en trabajo parcial, a lo largo del territorio español se siguen trabajando más horas por trabajador y trabajadora, en línea con la media europea, a pesar de la elevada tasa de paro (Tabla 1).

Tabla 1. Redistribución de las horas trabajadas en España a los promedios holandeses y alemanes

\begin{tabular}{|c|c|c|c|c|c|c|}
\hline & $\begin{array}{c}\text { Horas anuales } \\
\text { trabajadas por } \\
\text { trabajador/a } \\
(2015)\end{array}$ & Empleos & $\begin{array}{l}\text { Total horas } \\
\text { trabajadas }\end{array}$ & Población activa & $\begin{array}{l}\text { Población en } \\
\text { desempleo }\end{array}$ & $\begin{array}{l}\text { Tasa de paro } \\
\text { (\%) }\end{array}$ \\
\hline España EPA $(3 T, 2016)$ & 1.691 & 18.527 .500 & 31.330 .002 .500 & 22.848 .300 & 4.320 .800 & 18,9 \\
\hline $\begin{array}{l}\text { Tasa de paro en los Países } \\
\text { Bajos }(2 \mathrm{~T}, 2016)\end{array}$ & - & - & - & - & - & 6,2 \\
\hline $\begin{array}{l}\text { Tasa de paro en Alemania } \\
(2 \mathrm{~T}, 2016)\end{array}$ & - & - & - & - & - & 4,2 \\
\hline $\begin{array}{l}\text { España a jornada del } \\
\text { año 2000, trabajando las } \\
\text { mismas horas en total y } \\
\text { manteniendo la población } \\
\text { activa }\end{array}$ & 1.753 & 17.872 .220 & 31.330 .002 .500 & 22.848 .300 & 4.976 .080 & 21,8 \\
\hline $\begin{array}{l}\text { España a jornada } \\
\text { neerlandesa }(-16,1 \%), \\
\text { trabajando las mismas horas } \\
\text { en total y manteniendo la } \\
\text { población activa }\end{array}$ & 1.419 & 22.078 .931 & 31.330 .002 .500 & 22.848 .300 & 769.369 & 3,4 \\
\hline $\begin{array}{l}\text { España a jornada alemana } \\
(-18,9 \%) \text {, trabajando las } \\
\text { mismas horas en total y } \\
\text { manteniendo la población } \\
\text { activa }\end{array}$ & 1.371 & 22.851 .935 & 31.330 .002 .500 & 22.848 .300 & -3.635 & 0,0 \\
\hline
\end{tabular}

EPA: Encuesta de Población Activa. 2T: segundo trimestre. 3T: tercer trimestre.

Fuente: Torrens y González de Molina (2016), con datos del Instituto Nacional de Estadística, Eurostat y la Organización para la Cooperación y el Desarrollo Económico.

horas de trabajo en los empleos de todas clases (Organización Internacional del Trabajo, 2011).

${ }^{20}$ Según aparece en el Informe de la Organización Internacional del Trabajo (2011: 14-15), realizado para el debate de la Reunión Tripartita de Expertos sobre la Ordenación del Tiempo de Trabajo.

${ }^{21}$ Véase el interesante artículo escrito por De la Fuente y Zubiri (2016: 102), que observan:

Un balance positivo de esta experiencia, considerada una referencia en la conquista de mayores cotas de productividad 'en especie' vía la RTT [reducción de tiempos de trabajo]. Estando cualquier experiencia plagada de contradicciones, podemos afirmar que las Leyes Aubry dan buena fe de la vigencia histórica de las políticas de RTT a favor de los trabajadores y de un reparto más justo de los excedentes productivos y sus mejoras técnicas y organizativas permanentes. Así mismo, se considera que la reducción generalizada, sustantiva, equitativa y redistributiva del tiempo de trabajo [...] es la única vía creíble para afrontar la ineludible reorganización de los trabajos reproductivos y de cuidados, en la línea de su reparto consistente entre hombres y mujeres.
${ }^{22}$ Así, en la Unión Europea ha tenido lugar un importante crecimiento de la involuntariedad del tiempo parcial y del trabajo temporal (European Trade Union Institute, 2015: 30). 
Entre las medidas voluntarias, la más importante es permitir el cambio voluntario y reversible de duración de jornada por parte del trabajador o trabajadora (y de obligada aceptación por la empresa, salvo fuerza mayor). El caso neerlandés es paradigmático en este sentido, pues combina una legislación que permite que las/os trabajadores/as opten libremente por cambiar de tipo de jornada, y de manera reversible, con garantía de que se mantienen las condiciones laborales entre los trabajos a tiempo completo y reducido. Ello hace que los Países Bajos tengan la mayor tasa de trabajo a tiempo parcial del mundo y cuenten también con una gran mayoría de trabajadoras/es que trabajan a tiempo parcial de forma voluntaria (Visser et al., 2004).

A nuestro parecer, la reducción legal de la jornada laboral a 35 horas semanales, acompañada de la incentivación de otras reducciones voluntarias, es la principal garantía para la obtención de un empleo.

\section{La renta básica universal como derecho subjetivo}

La renta básica no es simplemente una herramienta de redistribución de la riqueza o de lucha contra la pobreza, sino que es también un modo de garantizar la existencia material de cada persona, y de que el conjunto de la sociedad pueda ser más libre, pues, según el concepto aprobado por la Basic Income Earth Network, que es la red internacional que trabaja a favor de la implementación de la renta básica, ésta se traduce en un pago periódico, individual e intransferible. Es universal e incondicional, es decir: se le distribuye a toda la población, independientemente de la edad, la raza, el género, el origen, o la condición socioeconómica y laboral. No está atada a ningún estatus económico o laboral, ni a un determinado nivel de renta ${ }^{23}$.

\subsection{Repartir lo que es de todas las personas}

Es importante reflexionar sobre que las riquezas acumuladas y los beneficios privados han sido logrados con el esfuerzo de todas las personas. Mariela Mazzucato (2014) nos explica cómo la innovación tecnológica, en lo que se refiere a la creación de internet, el sistema GPS, las pantallas táctiles, los dispositivos de grabación, los buscadores de algoritmos o los distintos tipos de baterías, ha sido el resultado de una financiación gubernamental a lo largo de varias décadas, y por consiguiente, de un esfuerzo colectivo por parte de la sociedad, a través de sus impuestos. Sin embargo, el resultado de la socialización de los costes ha derivado en la privatización de los beneficios. En teoría, dicha financiación se devuelve al Gobierno y a la comunidad mediante los impuestos corporativos, $\mathrm{me} / \mathrm{s}$. pero en la práctica sabemos que muchas veces no es así, pues los paraísos fiscales, la evasión de impuestos, así como la bajada de impuestos a empresas lo evitan. El patrón de privatización de los beneficios y socialización de los costes ocurre en la innovación tecnológica, pero también en el sector bancario y financiero, así como en la crisis económica más reciente, en la cual las personas ricas se han hecho más ricas, pero los costes se han facturado a la sociedad.

\subsection{Terminar con la pobreza}

La renta básica, con la gran redistribución de riqueza que provoca, permite combatir eficazmente la pobreza, que no es sólo privación y carencia material, habida cuenta de que el importe de la renta básica debe proporcionar un estándar de vida decente, equivalente al menos al umbral de la pobreza. En otras palabras, permite acabar aquí y ahora con una situación estructural de falta de ingresos que viola de forma permanente el derecho humano más elemental de millones de personas, pues afecta gravemente a su vida y libertad. Además, permite garantizar el derecho a una vida digna es una obligación jurídica exigible a los poderes públicos ${ }^{24}$.

${ }^{24}$ El Pacto Internacional de los Derechos Económicos, Sociales y Culturales de 1966 -en lo sucesivo, PIDESC-, que forma parte del ordenamiento interno español tras haber sido ratificado por España (BOE, $\mathrm{n}-103,30-4-77$ ), incluye, entre otros, los derechos a la vivienda, a la salud física y mental, y a la educación. Además, España ratificó en septiembre de 2010 el Protocolo Facultativo de dicho Pacto, lo que permitirá la interposición de quejas individuales por vulneración de alguno de los derechos proclamados.

Entre otros artículos del PIDESC, cabe destacar el 11.1, que reconoce "el derecho de toda persona a un nivel de vida adecuado para sí y su familia, incluso alimentación, vestido y vivienda adecuados, y a una mejora continua de las condiciones de existencia". A este respecto, a España se le recomendó:

Garantizar que todas las medidas de austeridad adoptadas identifiquen el contenido mínimo esencial de todos los derechos del Pacto, y tomar todas las medidas apropiadas para proteger este contenido esencial en cualquier circunstancia, especialmente para las personas y los grupos desfavorecidos y marginados" (Comité Internacional de los Derechos Económicos, Sociales y Culturales, en lo sucesivo Comité DESC, 2012).

Y conforme al artículo 2.1 del PIDESC, "cada uno de los Estados Partes en el presente Pacto se compromete a adoptar medidas, tanto por separado como mediante la asistencia y la cooperación internacionales, especialmente económicas y técnicas, hasta el máximo de los recursos de que disponga, para lograr progresivamente, por todos los medios apropiados, inclusive en particular la adopción de medidas legislativas, la plena efectividad de los derechos aquí reconocidos". La expresión "por todos los medios apropiados" debe ser interpretada en un sentido amplio, y dado que son los propios poderes públicos los que cuentan con la información relevante, les corresponde determinar qué medios son más apropiados para realizar progresivamente estos derechos (Comité DESC, 1990). Esto significa que la administración pública debe asumir la carga de la prueba y demostrar que las medidas y políticas adoptadas son las idóneas para garantizar un adecuado nivel de cumplimiento de los derechos socioeconómicos. Asimismo, el carácter apropiado de un instrumento depende, en última instancia, de su eficacia para la materialización de los derechos sociales (Comité DESC, 1998). 


\subsection{Evitar la trampa de la pobreza}

Además, la renta básica acabaría con la pobreza de un modo eficaz, algo que otras medidas no pueden hacer. Al ser incondicional, no desincentiva el trabajo, al contrario de lo que acontece con las ayudas sociales actuales, ya que quien opta a un empleo, se arriesga a dejar de recibirlas. Muchas veces el nivel salarial no compensa frente al de la ayuda, pues hay que tener en cuenta factores como el coste de transporte y la reducción del tiempo que podemos dedicarle a la familia o al cuidado de personas. Así, muchas personas con dichas ayudas acaban por no optar por un empleo, lo cual hace que caigan en la trampa de la pobreza. Dicha trampa no sólo se evita con la renta básica universal, ya que ésta no se perdería si se recomienza la actividad laboral, sino que además, la incondicionalidad sería especialmente beneficiosa para aquellas personas también en situación de pobreza, pero que, debido a las estrictas condiciones de las ayudas actuales y a la burocracia, no reciben ayudas, y por tanto, tampoco son capaces de sobrepasar el umbral de la pobreza. Así, la renta básica permitiría que nadie cayese en estos huecos administrativos.

\subsection{Invertir las relaciones de poder, que perpetúan las desigualdades sociales}

Junto con este mayor efecto redistributivo, la incondicionalidad de la renta básica tiene el potencial de invertir las relaciones persona empleada-persona empleadora, trabajo-capital. Es a través de la desvinculación de la supervivencia y existencia material del empleo que se puede generar un proceso liberalizador para las personas, pues permite vivir sin ataduras al empleo, que en gran medida es precario y poco realizador, siendo condicionado y marcado por el mercado laboral, que está gestionado por las grandes potencias y élites económicas, que priorizan los beneficios y dejan al margen la dignidad y la justicia social. Por tanto, al introducir la renta básica las personas no sólo son capaces de desvincularse de su empleo, sino también de una forma de vida condicionada por las reglas que imponen el mercado y las grandes multinacionales. Estas últimas definen las condiciones laborales y salarios de la mayoría de personas, es decir, definen sus vidas, su poder adquisitivo, en qué invierten su tiempo y quiénes son. Esto no es sólo altamente peligroso por el grado de descontrol que las personas podamos ejercer sobre nuestras condiciones vitales, sino que es profundamente antidemocrático. Hoy en día, en España tanto como en el País Vasco ${ }^{25}$ se ha alcanzado tal nivel de riqueza y avance tecnológico que es posible permitirse trabajar menos horas y repartir

${ }^{25}$ En el artículo “¿Necesita la Comunidad Autónoma Vasca una Renta Básica Universal? Sí, por supuesto. Y más allá”, escrito por Arcarons, Raventós y Torrens (2017), se explica que el coste de una renta básica de 7.902 euros por adulto y el $20 \%$ para cada menor y para toda la población de la CAV sería de $14.800,4$ millones de euros, lo cual es perfectamente financiable. los enormes beneficios de una manera más justa, que nos permita desvincularnos de la dictadura de las grandes corporaciones. Es importante y urgente hacerlo cuanto antes, porque las multinacionales se rigen por la generación de máximos beneficios y no están para solventar problemas sociales.

\subsection{Mejorar las estructuras de poder en parejas y familias}

El modelo socialmente aceptable de pareja que se ha impuesto en la historia ha sido uno basado en un hombre y una mujer heterosexuales, que conviven en una misma residencia. Este modelo, junto con otras dinámicas de desigualdad laboral, institucional y social de la mujer, ha dado como resultado que se adopten roles de género diferenciados. Así, pese a la incorporación de la mujer en el mercado laboral, la reciente crisis económica ha hecho que se retroceda de nuevo hacia el hombre trabajador y la mujer como ama de casa. Esta estructura familiar, social y económicamente impuesta ha sido un buen caldo de cultivo - aunque no por ello la causa-de la violencia de genero doméstica, así como de relaciones de pareja infelices, amén de incapacitar para un pleno desarrollo personal y profesional de la mujer. Al estar los hombres económicamente mejor remunerados, éstos han tenido también un mayor poder de negociación en el seno de las relaciones de pareja.

El componente económico es un factor decisivo que atrapa a las mujeres en la esfera doméstica de la pareja con la que conviven. La renta básica, tiene el potencial de abordar y mejorar notablemente esta situación. Para empezar, al recibirla individualmente tanto hombres como mujeres - algo que no sucede con las ayudas tradicionales-, pone a ambos miembros de la pareja en una situación de igualdad económica de base. Así, mejora notablemente el poder de negociación de la mujer, situándola en posición de igualdad frente al hombre. Además, dota de libertad económica a ambos miembros de la pareja, permitiéndoles establecer una vida autónoma, sin necesidad de economía de escala con una pareja. Ésta es la salida de emergencia para las mujeres que sufren violencia de género, pero también es una ayuda fundamental para las personas que son infelices en sus parejas.

\subsection{Permitir la calidad de vida de la juventud, que es el futuro}

Pero, además, hay otras virtualidades de la renta básica que debemos destacar ${ }^{26}$. Entre ellas, los potenciales beneficios para la juventud, sobre todo en el Estado español, donde la tasa de emancipación

${ }^{26}$ En "Algunas virtualidades de la renta básica”, publicado en el Boletín Hegoa, no 48 de octubre de 2016, comentamos otros ámbitos de interés para la renta básica como la igualdad de género, la democracia y la participación ciudadana o la eficiencia de la Administración, además de evitar el estigma a quienes perciben ayudas sociales. 
juvenil, de un $46 \%$ (entre 18 y 34 años), es la más baja de la UE, a pesar de que el derecho a una vivienda está recogido en el artículo 47 de la Constitución española. Pero éste es uno de los muchos motivos por los cuales una renta básica es indispensable para la juventud. El actual mercado laboral es muy diferente al de hace unos años, y la mayoría de la juventud, cada vez más preparada, tiene más dificultades para encontrar su hueco en él, ya que está marcado por los cambios tecnológicos, la globalización y por la precarización del empleo. Más que nunca, las ayudas para el emprendimiento son necesarias para que la juventud pueda utilizar sus conocimientos y capacidades en un trabajo adecuado, y que no se vea obligada a emigrar o a conformarse con empleos muy precarios.

\subsection{Conciliar frente al mercado laboral y vivir una vida creativa}

Son muchas las personas que sostienen la tesis de que no volveremos a ver niveles de empleo similares a los de la década de 1950. Debemos, por tanto, abandonar esta lógica de trabajo entendida como un empleo en el mercado laboral, la lógica de formarse y ocupar un puesto predefinido en este mercado. Además, la participación en una economía internacional hace que cada país genere distintas demandas, como sucede, por ejemplo, en el sector hostelero. En este contexto de nuevos cambios y una demanda específica, pero una educación y preparación inadaptada a la actividad económica, una renta básica se presenta como el primer colchón frente a la precariedad laboral y personal. La renta básica permitiría un espacio temporal en el que las personas pudiesen desarrollar sus capacidades al máximo, encontrar su contribución a la sociedad y emprender nuevos e innovadores proyectos que beneficien a la sociedad. Este proceso parte de la lógica creativa del ser humano y de su capacidad intelectual, humana y artística de descubrir las potencialidades que le puede ofrecer a la sociedad en la que vive.

\subsection{Desterrar la renta básica como una panacea}

Se ha señalado extensivamente, tanto entre personas defensoras como críticas a la renta básica, que ésta no es una panacea, pues sólo es una medida y no toda una política económica. Una vez reconocido este punto, cabe destacar que pocas políticas son capaces de abordar una serie de contextos y problemas sociales, como la violencia de género, la trampa de la pobreza, el desempleo tecnológico o la adaptación a un mercado laboral en el que las carreras universitarias no han sabido adaptarse, por citar algunos ejemplos. La renta básica consigue, al ofrecer una mejora consistente en estos contextos tan centrales a la exclusión social, fortalecer significativamente las perspectivas de inclusión. En una primera instancia, por tanto, la renta básica consigue facilitar varios escalones a la inclusión social. En una segunda instancia, y como política a largo plazo, permanente y de futuro, consigue prevenir la exclusión social, porque está diseñada para conseguir los derechos de universalidad e incondicionalidad, y de una vida digna al margen de la participación en el mercado.

En definitiva, el reconocimiento del derecho universal a la renta básica debe ser un instrumento fundamental de cualquier plan de inserción activa.

\section{Educación inclusiva}

Todo ser humano es limitado, pero con gran potencialidad. Por ello, es necesario que, en el proceso educativo, se desarrollen las competencias según nuestras capacidades, de manera que se consiga una alta motivación y vivencias de éxito, que favorezcan una adecuada autoestima. Esto implica un proyecto educativo en el que no haya que elegir entre convivencia y aprendizaje instrumental, con una pedagogía de máximos que posibilite espacios libres de violencia y solidarios donde se trabaje en función de altas expectativas y en todos los ámbitos -cognitivo, académico, afectivo y social-; y siempre con la mirada en un futuro esperanzador con posibilidades reales de libre elección para todas y $\operatorname{todos}^{27}$. Sin duda, ello exige muchísimo trabajo en el profesorado, en las familias y en el voluntariado, y aprendizaje del máximo nivel en el alumnado, con un diálogo igualitario, donde estén bien presentes la ilusión y la solidaridad ${ }^{28}$.

En la comunidad educativa vasca ${ }^{29}$, varios colectivos vienen trabajando desde hace años en clave de comunidades de aprendizaje, pudiendo mencionarse como ejemplo ilustrativo el del colegio público de Elejabarri, donde un alumnado con alto porcentaje de procedencia inmigrante y gitana, que tenía un muy elevado índice de fracaso escolar, fue motivado

${ }^{27}$ Al hablar de la educación inclusiva, un referente ineludible en el ámbito internacional es la Conferencia Mundial de Jomtien, celebrada en 1990, sobre educación para todos, o el proyecto europeo INCLUD-ED: Strategies for Inclusion and Social Cohesion in Europe from Education (2006-2011), que es el mayor sobre educación obligatoria financiado bajo la prioridad 7, Citizens and Governance in the Knowledge-based Society, del Sexto Programa Marco de la Comisión Europea, que ha analizado las estrategias educativas que se llevan a cabo dentro de las escuelas y que conducen a la exclusión social, a fin de aportar actuaciones que contribuyan al éxito educativo y a la cohesión social. Igualmente son numerosas las aportaciones bibliográficas, aunque únicamente citaremos las de Echeita y Sandoval (2002), Flecha, Padrós y Puigdellívol (2003), Sarto y Venegas (2009), Martínez Abellán, De Haro y Escarbajal (2010) o el citado proyecto europeo INCLUD-ED, del que hace una completa recesión Serrano (2015).

${ }^{28}$ Y también "iAquí hay mucho amor!", tal como transmitía una madre en los pasillos en el encuentro celebrado en el País Vasco, el 10 de mayo de 2017, por 17 docentes de centros públicos y concertados de Cantabria y de todas las etapas educativas (Educación Inclusiva, 2017b).

${ }_{29} \mathrm{El} 17$ de mayo de 2017 se iniciaron en Bilbao las Jornadas sobre Actuaciones Educativas de Éxito en la Escuela Inclusiva (Educación Inclusiva, 2017a). Ello es consecuencia de que en Euskadi hay equipos educativos que son verdaderas comunidades de aprendizaje, como sucede, entre otros, en los centros de educación infantil y primaria lbaibe y Elejabarri, así como el instituto de educación secundaria Artabe. 
gracias a un acompañamiento individualizado basado en los puntos fuertes de esas niñas y niños ${ }^{30}$.

\section{A modo de conclusiones}

En este artículo, hemos defendido que cualquier política de inclusión debe cuestionar las estructuras y factores que acentúan la vulnerabilidad de las personas de la comunidad, en vez de poner su principal atención en las consecuencias que acarrea a las personas afectadas. Por ello, hemos planteado otro modelo de economía, concretamente una social y solidaria.

Al referirnos al empleo como instrumento clave en la inclusión, hemos denunciado que se desarrolla en condiciones laborales de precariedad, y hemos propugnado como alternativa la reducción de la jornada laboral.

Otro concepto central ha sido el de la riqueza: disponemos de mucha riqueza, pero está mal e injustamente repartida. Esta situación se está exacerbando con el tiempo, debido a que así lo están permitiendo las dinámicas de mercado, las elites económicas y las instituciones y poderes políticos. Como medida innovadora, se ha propuesto la renta básica. Se han mostrado las utilidades y potencialidades de esta medida, no como una panacea, sino como una escalera que nos permita, en una variedad de contextos y problemas, mejorar significativamente la inclusión social. Hemos querido destacar que no se trata de un parche, sino de una medida que a largo plazo puede resultar ideal para prevenir la exclusión social, ya que está basada en los pilares de universalidad e incondicionalidad.

Como último punto, hemos señalado que la educación inclusiva debe ser un elemento fundamental para el progreso individual y colectivo de todas las personas, para lo que es necesario que la metodología educativa se base en las potencialidades de cada una de ellas. 
ALBA, M.; y RUIZ, S. (2016): Beneficios para quién. Los paraísos fiscales como principal amenaza para una fiscalidad justa, serie Informes, $\mathrm{n}$ 38, Oxfam Intermon ['https://oxfamintermon. s3.amazonaws.com/sites/default/files/ documentos/files/Beneficios-para-quien.pdf`].

ALONSO, J. I.; y MARCOS, L. (2017): Desheredados. Desigualdad infantil, igualdad de oportunidades y políticas públicas en España. Anexo Euskadi, Save The Children España [<https://www.savethechildren.es/sites/ default/files/imce/docs/save_the_childrenanexo_vasco_espanaol_web.pdf $)$ ].

ARCARONS, J.; RAVENTÓS, D.; y TORRENS, L. (2017): “¿Necesita la Comunidad Autónoma Vasca una Renta Básica Universal? Sí, por supuesto. Y más allá”, Sin Permiso, 29-1-17 [<http://www. redrentabasica.org/rb/necesita-la-comunidadautonoma-vasca-una-renta-basica-universal-sipor-supuesto-y-mas-alla/>].

CARRASQUER, P. (2009): “La doble presencia. El trabajo y el empleo femenino en las sociedades contemporáneas" [tesis doctoral], Barcelona, Universidad Autónoma de Barcelona [rhttps:// ddd.uab.cat/pub/tesis/2009/tdx-0406110152629/pco1de1.pdf>].

CARRASQUER, P. et al. (1998): “El trabajo reproductivo”, Papers, № 55, págs. 95-114 [<http:// www.raco.cat/index.php/papers/article/ viewFile/25507/25340>].

CASTRILLÓN, V. et al. (2015): “Propuestas de reducción del tiempo de trabajo en Gipuzkoa", Lan Harremanak, no 34 [khttp://www.ehu.eus/ ojs/index.php/Lan_Harremanak/article/ view/16568/14760〉].

COMISIÓN EUROPEA (2016): “Dictamen del Comité Económico y Social Europeo sobre 'Los efectos de la digitalización sobre el sector de los servicios y el empleo en el marco de las transformaciones industriales' (Dictamen exploratorio)", Diario Oficial de la Unión Europea, 5-1-16, 2016/C 013/24 [<http:// eur-lex.europa.eu/legal-content/ES/ TXT/?uri=CELEX:52015AE0765>].

COMITÉ INTERNACIONAL DE LOS DERECHOS ECONÓMICOS, SOCIALES Y CULTURALES (2012): Observaciones Finales: España, E/C.12/ESP/Co/5, 18-5-12, § 8 [khttp://www.derechoshumanos.net/ONU/ ComiteDerechosEconomicosSocialesCultural es-CESCR.htm>].

- (1998): Observación General 9 9 . La aplicación interna del Pacto, E/C.12/1998/24, 3-12-98, § 5 [<http:// www.derechoshumanos.net/ONU/ComiteDer echosEconomicosSocialesCulturales-CESCR. $\mathrm{htm}\rangle$.

EDUCACIÓN INCLUSIVA (2017a): “ADARRA-Jornadas sobre Actuaciones Educativas de Éxito en la Escuela Inclusiva", 23-5-17 [rhttps:// metodologiainclusiva.wordpress. com/2017/05/23/adarra-jornadas-sobreactuaciones-educativas-de-exito-en-la-escuelainclusiva/>].

- (2017b): "Encuentro con Comunidades de Aprendizaje en Euskadi”, 10-5-17 [<https:// metodologiainclusiva.wordpress. com/2017/05/10/encuentro-con-comunidadesde-aprendizaje-en-euskadi>].

CONFEBASK (2016): Informe de Coyuntura Económica. Principales rasgos económicos de 2016 y perspectivas 2017, Bilbao, Confebask [ [rhttp:// www.confebask.es/sites/default/files/varios/ informe-coyuntura-economica-2016-2017.pdf〉].

DE LA FUENTE, M.; y ZUBIRI, J. B. (2016): “Efectos sobre el empleo de la reducción del tiempo de trabajo: 
aprendizajes de la experiencia francesa", Lan Harremanak, no 34 , págs. 79-105, [<http://www. ehu.eus/ojs/index.php/Lan_Harremanak/ article/view/16558>].

DE LA RICA, S.; DEMEL, S.; y MILLER, L. (2017): Informe Laboral Euskadi. Abril-junio 2017, Universidad del País Vasco UPV-EHU (Unidad de Formación e Investigación BETS) [<http://www.eapmaster. org/informelaboraleuskadi/Analisis_

PaisVasco_2trim2017_web.pdf/>].

DEPARTAMENTO DE EMPLEO Y POLÍTICAS SOCIALES. ÓRGANO ESTADÍSTICO ESPECÍFICO (2014): Encuesta de Necesidades Sociales 2014, Módulo EPDS-Pobreza. Comunidad Autónoma de Euskadi. Principales resultados / Behar Sozialei buruzko Inkesta 2014 PGDI PobreziaModulua. Euskal Autonomia Erkidegoa. Emaitza nagusiak, Vitoria-Gasteiz, Eusko JaurlaritzaGobierno Vasco [<http://www.gizartelan.ejgv. euskadi.net/contenidos/informe_estudio/ informe_epds_2014/es_epds2012/adjuntos/ Informe\%20M\%C3\%B3dulo\%20EPDS\%20 2014_es.pdf>].

DEPARTAMENTO DE HACIENDA Y ECONOMÍA (2016): "Informe sobre economía sumergida y fraude fiscal en Euskadi” [nota de prensa], Eusko Jaurlaritza-Gobierno Vasco [<http://www. euskadi.eus/webo1-s20ga/es/contenidos/ noticia/2016_06_09_33321/es_33321/33321. htmls].

ECHEITA, G.; y SANDOVAL, M. (2002): "Educación inclusiva o educación sin exclusiones", Revista de Educación, no- 327, págs. 31-48, [<https:// metodologiainclusiva.files.wordpress. com/2016/02/educacion-inclusiva-oeducacicзb3n-son-exclusiones.pdf $>$ ].

ELÍAS, A.; y RINCÓN, L. (2016): “Algunas virtualidades de la renta básica”, Boletín Hegoa, no- 48 [<http:// boletin.hegoa.ehu.es/mail/45'].

ESPAÑA (1977): “Instrumento de Ratificación de España del Pacto Internacional de Derechos Económicos, Sociales y Culturales, hecho en Nueva York el 19 de diciembre de 1966", Boletín Oficial del Estado, no 103 , 30-4-77, págs.9.3439.347 [ [hhttps://www.boe.es/buscar/doc. php?id=BOE-A-1977-10734'].

EUROPEAN TRADE UNION INSTITUTE (2015): Benchmarking Working Europe, Bruselas, European Trade Union Institute.

FELGUEROSO, F. (2014): "Midiendo el paro como los americanos: aún más líderes", Nada es Gratis [<http://nadaesgratis.es/felgueroso/ subempleo-y-precariedad-indicadores-para-larecuperaci\% $\left.\mathrm{C}_{3} \% \mathrm{~B} 3 n>\right]$.

FLECHA, R.; PADRÓS, M.; y PUIGDELLÍVOL, I. (2003): "Comunidades de aprendizaje: transformar la organización escolar al servicio de la comunidad", Organización y Gestión Educativa. Revista del Fórum Europeo de Administradores de la Educación, $\mathrm{n}{ }^{\circ}$ 5, págs. 4-8.

FORO ECONÓMICO MUNDIAL (2016): The Future of Jobs. Employment, Skills and Workforce Strategy for the Fourth Industrial Revolution, Cologny, Foro Económico Mundial [rhttp://www3.weforum. org/docs/WEF_Future_of_Jobs.pdf〉].

GONZÁLEZ BILBAO, J. G. B. (2014): “Elejabarri Eskola: eraldaketaren bila” [presentación y vídeo] [<https://prezi.com/_7pne7rbwx33/ceipelejabarri-hlhi-upv-ehu/>].

INSTITUTO MANU ROBLES-ARANGIZ (2017): Análisis de coyuntura, $\mathrm{n}-134$, Instituto Manu RoblesArangiz [shttps://www.mrafundazioa.eus/ es/centro-de-documentacion/analisis-decoyuntura/analisis-de-coyuntura-134'].

MACÍAS, I.; y RUIZ RODRÍGUEZ (2017): Una economía para el 99\%: España, un crecimiento económico que deja fuera a las personas vulnerables, serie Informes, no 42, Oxfam-Intermón [«https:// www.oxfamintermon.org/sites/default/files/ documentos/files/Informe-Una-economia-para99-espana-oxfam-intermon.pdf〉].

MARTíNEZ ABELLÁN, R.; DE HARO, R.; y ESCARBAJAL (2010): "Una aproximación a la educación inclusiva en España", Revista Educación Inclusiva, $\mathrm{n}$ - 1, págs. 149-164 [<http://www.ujaen.es/revista/ rei/linked/documentos/documentos/5-9.pdfs].

MARX, K. (1866): “Instrucción sobre diversos problemas a los delegados del Consejo Central Provisional”, Obras escogidas, tomo II, Moscú, Editorial Progreso [<https://www.marxists.org/espanol/ $\mathrm{m}-\mathrm{e} / 1860 \mathrm{~s} /$ isdp66s.htm〉].

- (1864): “Manifiesto Inaugural de la Asociación Internacional de los Trabajadores" [publicado en inglés en el folleto Addres and Provisional Rules of the Working Men's International Association, Established September 28, 1864, at a Public Meeting held at St. Martin's Hall, Long Acre, London] [<https://www.marxists. org/espanol/m-e/186os/1864fait.htm〉].

MAZZUCATO, M. (2014): El Estado emprendedor, RBA Libros.

MONTES, P. (1996): Golpe de Estado al bienestar, Barcelona, Icaria.

OPINNO (2016): Informe Adecco sobre el futuro del trabajo en España, Pozuelo de Alarcón, Adecco.

ORGANIZACIÓN INTERNACIONAL DEL TRABAJO (2011): El tiempo de trabajo en el siglo XXI. Informe para el debate de la Reunión tripartita de expertos sobre la ordenación del tiempo de trabajo (1721 de octubre de 2011), Ginebra, Organización Internacional del Trabajo [<http://www.ilo. org/wcmsp5/groups/public/---ed_protect/--protrav/---travail/documents/publication/ wcms_162148.pdf〉].

RAVENTÓS, D.; y WARK, J. (2016): “Trabajo remunerado, trabajo doméstico, trabajo voluntario y Renta Básica”, Sin Permiso, 8-5-16 [ [<http://www. sinpermiso.info/textos/trabajo-remuneradotrabajo-domestico-trabajo-voluntario-y-rentabasica〉].

RED DE REDES DE ECONOMÍA ALTERNATIVA Y SOLIDARIA (2011): Carta de Principios de la Economía Solidaria, Red de Redes de Economía Alternativa y Solidaria [<http://www. economiasolidaria.org/carta.php〉].

SARTO, M. P.; y VENEGAS, M. E. (2009): Aspectos clave de la educación inclusiva, colección Investigación, Salamanca, INICO [<http://sid.usal.es/idocs/ F8/FDO22224/educacion-inclusiva.pdf>].

SERRANO, M. A. (2015): “Flecha, R. (ed.) (2015). Successful educational actions for inclusion and social cohesion in Europe. Berlin: Springer" [reseña], Revista Internacional de Organizaciones, $\mathrm{n}-14$, 
págs. 161-164 [rhttp://www.revista-rio.org/ index.php?journal=revista_rio\&page $=$ article\& op=download\&path $\% 5$ B $\% 5 \mathrm{D}=183$ \& path $\% 5 \mathrm{~B} \%$ $5 \mathrm{D}=168>$.

SOTO, A. (1989): El trabajo industrial en la España Contemporánea (1874-1936), Barcelona, Anthropos.

TAX JUSTICE NETWORK (2016): "Size of the problems" [<http://www.taxjustice.net/topics/more/sizeof-the-problem/>].

TORRENS, L.; y GONZÁLEZ DE MOLINA, E. (2016): “La garantía del tiempo libre: desempleo, robotización y reducción de la jornada laboral (parte 2)", Sin Permiso, 12-12-16 [<http://www. redrentabasica.org/rb/la-garantia-del-tiempolibre-desempleo-robotizacion-y-reduccion-dela-jornada-laboral-parte-2/>].

VISSER, J., et al. (2004): "The Netherlands: from atypicality to typicality", en SCIARRA, S.; DAVIES, P.; y FREEDLAND M. (eds.), Employment Policy and the Regulation of Part-time Work in the European Union: A Comparative Analysis, Cambridge, Cambridge University Press, págs. 190-222 [<https://dare.uva.nl/ document/2/143586>]. 